Itinéraires Itinéraires

Littérature, textes, cultures

\title{
Uncreative Writing, Close Reading ? À propos de Vanessa Place et Kenneth Goldsmith
}

Uncreative Writing, Close Reading? About Vanessa Place and Kenneth Goldsmith

Michel Murat

\section{OpenEdition}

Journals

Édition électronique

URL : http://journals.openedition.org/itineraires/3961

DOI : $10.4000 /$ itineraires.3961

ISSN : 2427-920X

Éditeur

Pléiade

Référence électronique

Michel Murat, « Uncreative Writing, Close Reading ? À propos de Vanessa Place et Kenneth Goldsmith », Itinéraires [En ligne], 2017-3 | 2018, mis en ligne le 15 juin 2018, consulté le 02 mai 2019. URL http://journals.openedition.org/itineraires/3961; DOI : 10.4000/itineraires.3961

Ce document a été généré automatiquement le 2 mai 2019.

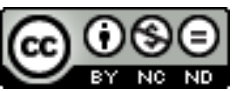

Itinéraires est mis à disposition selon les termes de la licence Creative Commons Attribution - Pas d'Utilisation Commerciale - Pas de Modification 4.0 International. 


\section{Uncreative Writing, Close Reading? À propos de Vanessa Place et Kenneth Goldsmith}

Uncreative Writing, Close Reading? About Vanessa Place and Kenneth Goldsmith Michel Murat

1 La poésie conceptuelle est une des plus remarquables manifestations actuelles des avantgardes américaines. En prenant l'exemple de deux de ses représentants majeurs, Kenneth Goldsmith et Vanessa Place, je me propose ici trois sujets de réflexion ${ }^{1}$ : la relation, filiation ou rupture, de cette poésie avec la tradition moderniste ; les implications sociales et politiques du geste d'appropriation de discours qui la constitue; la possibilité de considérer les œuvres issues de ces pratiques «non créatives» comme des textes susceptibles non seulement d'une compréhension conceptuelle, mais d'une lecture attentive à la forme, telle que le close reading en a posé les principes.

\section{« No more retinal poetry »}

2 Je prendrai comme point de départ la proposition faite par la revue Poetry de célébrer le centenaire du manifeste d'Ezra Pound, "A Few Don'ts by an Imagiste », qu'elle avait publié en mars 1913, par une nouvelle série de prescriptions négatives, «A Few More Don'ts ». Quatre poètes s'y sont livrés, deux femmes et deux hommes, un blanc et un noir. En tête figure un texte de Vanessa Place, « No more » :

No more lines on the luminescence of light, of whatever variation.

No more elegies of youth or age, no polyglottal ventriloquism.

No more songs of raw emotion, forever overcooked.

No more the wisdom of banality, which should stay overlooked.

No more verbs of embroidery.

No more unintentional phallacy.

No more metaphor, no more simile. Let the thing be, concretely.

No more politics put politically: let the thing be concretely.

No more conditional set conditionally - let the thing be already. 
No more children pimped out to prove some pouting mortality.

No more death without dying - immediately.

No more poet-subject speaking into the poem-mirror, watching the mouth move,

fixing the thinning hair.

No more superiority of the interiority of that unnatural trinity - you, me, we - our

teeth touch only our tongues.

No more Gobstoppers: an epic isn't an epic for its fingerprints.

No more reversals of grammar if as emphasis.

No more nature less natural; no more impiety on bended knee.

No more jeu de mot, no more mot juste.

No more retinal poetry².

Voilà un manifeste dont l'énonciation a quelque chose de paradoxal : il refuse le mot juste tout en recourant à des expressions comme "polyglottal ventriloquism » ou " wisdom of banality»; le jeu de mots, alors qu'il est question d' « unintentional phallacy»; la métaphore, mais « pimped out » ou "Gobstoppers » (de très populaires bonbons durs) ne sont rien d'autre que des métaphores. La reformulation de « don't » en « no more » est la marque d'une conscience historisante et d'une orientation progressiste. Mais plusieurs strates de lieux communs se superposent, si bien qu'il est difficile de se situer dans cette histoire: Vanessa Place reformule le mot d'ordre de l'objectivisme ("let the thing be »), ce qui nous met dans les années 1930. Elle semble s'en prendre à la fois à un fond de romantisme (le poème miroir de la subjectivité), à des traits de la poésie victorienne (la broderie, la luminescence), mais aussi à Pound lui-même et à ses héritiers du Black Mountain College (le polyglottisme, l'épique), et à la poésie contestataire de la Beat Generation (la politique faite politiquement). La référence finale nous ramène à l'année 1913, où Marcel Duchamp dit avoir abandonné la " peinture rétinienne ». L'argument est ici que la poésie est en retard sur les arts plastiques, et qu'elle doit se mettre à l'heure nouvelle. Celle-ci n'est définie que par des prédicats assez vagues; ce qui serait pour la poésie l'équivalent de la peinture (non-) rétinienne demeure à l'état de présupposé. La poésie, comprenons-nous, doit faire sa révolution conceptuelle. Mais peut-on dire que pour ce qui la concerne, nous sommes encore en 1913 ?

Si l'on réfléchit aux motifs d'une telle intervention, la réponse est oui. On pourrait se demander si le texte donne parodiquement l'exemple de ce qu'il ne faut plus faire, en apportant une contribution sarcastique à une institution vénérable - un texte bon pour Poetry, avec ce qu'il faut de parallélismes et de rimes. Mais il est plus vraisemblable que Vanessa Place se serve de cette tribune pour attaquer les idées et les pratiques dominantes de la poésie américaine, en particulier celles qui sont transmises par les cours de creative writing. Considérés dans leur ensemble, ceux-ci véhiculent une idéologie de l'expression de soi, du développement personnel, du politiquement correct et de l'affirmation communautaire. Sur le plan formel ils transmettent un héritage routinisé soit de la tradition lyrique, soit d'un modernisme passé au filtre du New Criticism: typographie non conventionnelle, sensationnalisme trivial, obscurité de surface, alternance d'ironie pesante et de platitude foisonnent dans les textes qui se pressent aux portes, et souvent dans ceux qui franchissent ces portes ${ }^{3}$. Ces courants n'ont rien perdu de leur vigueur. Néanmoins l'avant-garde des language poets, après avoir été longtemps minoritaire et militante, est en voie d'institutionnalisation, et l'opposition entre mainstream et avant-garde commence à se déliter ${ }^{4}$. Quant à la poésie conceptuelle, elle s'est rapidement acquise, notamment grâce à Marjorie Perloff, une légitimité critique. La tribune de Poetry ne relève donc pas d'une prise de parole marginale, mais elle permet à 
Vanessa Place d'enfoncer le clou, et à la poésie conceptuelle de se poser comme la véritable avant-garde, celle du make it new d'aujourd'hui.

\section{Le langage de Statement of Facts}

5 L'auteur de ce poème s'est fait connaître en effet par une pratique radicale. Vanessa Place est avocate de son métier, spécialisée dans la défense d'hommes auteurs d'agressions sexuelles - un travail qui n'a rien de confortable d'un point de vue éthique. Son œuvre la plus connue, Statement of Facts (2010), est la transcription, sous des noms fictifs, de la partie narrative de dossiers d'appel qu'elle a elle-même rédigés ${ }^{5}$. Elle se situe dans la continuité directe de l'objectivisme de Reznikoff; mais avec Testimony, il y a une double différence: d'une part, l'implication de l'auteur dans l'affaire judiciaire elle-même (Reznikoff travaillait sur des archives jurisprudentielles, Place est partie prenante, et retranscrit sa propre rédaction); d'autre part la forme : Reznikoff récrit le texte en vers libres, et cette réénonciation lyrique qui en fait un recitative lui confère une puissante résonance historique et mémorielle. Place conserve la forme du texte juridique, sa segmentation, l'insertion de références documentaires; c'est une pratique neutre et plate, délibérément non poétique (de même que dans la performance elle use d'une voix non expressive, minimale, au point mort). Le texte n'est pas récrit mais simplement déplacé ou recadré. Et dans son style même il se distingue des archives utilisées par Reznikoff, dans lesquelles le récit - malgré une séquentialité rigoureuse - est fortement synthétique, construit selon les règles de ce que Musil appelait la «narration classique ». Chaque séquence de Testimony (du moins les plus frappantes d'entre elles) constitue un mythos tragique, nettement articulé, imposant le sentiment d'un destin: les circonstances, les caprices, les passions agissant sur le fond d'une nécessité commune qui est la réalité des États-Unis à un moment donné de leur histoire. Le style de Statement of Facts est au contraire celui d'un procès-verbal à la syntaxe répétitive, au vocabulaire restreint et technicisé :

The police arrived, Barbara B. was taken to the hospital and examined by a forensic nurse specialist, who took swabs from Barbara B.'s body, including an external genital swab, and swabs from Barbara B.'s breasts, and her right buttock. A reference blood sample and oral sample were collected in later dates. The samples were transported to the Long Beach police and then to the Los Angeles County Sheriff's Scientific Services Division. There were multiple bruises on Barbara B.'s leg; she had genital tears and a hematoma on her genitalia, indicative of sexual assault. The police kept Barbara B.'s nightgown. (RT 921, 1345-1352, 1403-1404, 1442-1444, 1466-14896)

6 L'intérêt immédiat de tels textes est de mettre en évidence une routine : le paragraphe que j'ai cité se retrouve, à peu près dans les mêmes termes, dans tous les statements, ce qui est normal puisqu'il s'agit d'un protocole médico-légal de prise en charge après agression. Mais les faits eux-mêmes se présentent avec la même allure routinière, dépassionnée, presque morne, si bien que la violence inhérente à ces scènes s'en trouve extraite comme par un procédé de déshydratation ou de lyophilisation. Le langage normalisé par les protocoles descriptifs, rapporté à une source énonciative unique (la déposition de la victime), sans écart possible, sans clivage ni modalisation, est réduit à lui-même. Il ne cherche pas à mettre sous les yeux : littéralement, il ne représente plus : 
The man fumbled, touching Barbara B.'s breasts with his hands and mouth, then put his penis in her vagina. She could not tell if he ejaculated or withdrew, but he put his penis on her vagina a second time; he also orally copulated her.

7 La lecture attentive d'un passage comme celui-ci nous fait mesurer à quel point l'usage d'un mot comme « viol» (qui dans ce contexte n'apparaît jamais) constitue à la fois une interprétation des faits et le point d'ancrage d'un ensemble de scénarios et de scènes mimétiques, nourri de textes et d'images - à quel point ce mot est littéraire. Mais ce n'est pas tout. Le texte est régi par un principe d'exhaustivité. Il reproduit sans les hiérarchiser, dans leur ordre de succession, tous les éléments d'une déposition donnée. Nombre de ces éléments détournent notre attention de ce qui nous semble le fait principal ; mais on pourrait aussi bien dire que le texte attire notre attention sur des comportements, gestes, paroles, qui sont aussi des faits :

[suite du texte précédent] Throughout, the man continue to tell Barbara B. he only wanted to make love to her and not to hurt her. After, the man told Barbara B. he was going to leave and she should count to fifty. She started counting to herself, he told her to count out loud. As Barbara B. heard the man leave, she asked him to close the door so her cats wouldn't get out; she heard him go to through the kitchen and close the sliding glass door as he left. Barbara B. then called police. (RT 917-920, 925)

Parler pour rassurer, compter jusqu'à cinquante comme à cache-cache, fermer la porte à cause des chats : nous voilà dans l'ordinaire, sans que ce mélange de l'ordinaire et du drame soit interprété - ni ironie, ni allégorie, ni «sagesse de la banalité »- ni même souligné. Joint à ce qu'on peut appeler l'assèchement rhétorique du drame, cet enregistrement exhaustif des circonstances a pour effet de rendre plus compliqué l'exercice du jugement moral, et c'est sans doute en cela que consiste le travail d'avocat de Vanessa Place. Dans cette phase du statement of facts il ne s'agit pas d'argumenter: le fait de refermer la porte de la cuisine n'est pas présenté comme une circonstance atténuante. Mais le simple compte rendu de ces gestes suffit à faire apparaître les agresseurs comme des êtres humains, égaux en droit à leurs victimes, et à nous rappeler que c'est sur ces bases que la justice doit faire son travail de qualification, de condamnation et de punition.

Mais la manière de performer de Vanessa Place produit de tout autres effets que la lecture du texte imprimé - des effets qui tendent à valoriser une saisie conceptuelle de l'œuvre, et la reversent au bénéfice de l'auteur. Sa performance de Statement of Facts est faite recto tono, à voix moyenne, avec un débit rapide et des moments presque mélopéiques, impression accentuée par le fait qu'elle s'accompagne d'un balancement régulier, sinusoïdal, du haut du corps ${ }^{7}$. Cette diction est anti-expressive, et si l'on peut formuler un jugement intuitif, anti-humaniste: elle ne laisse ni à la victime ni à l'agresseur la possibilité d'accéder, dans la conscience de l'auditeur, à un statut de personne à part entière. L'énoncé du cas, ponctué par les mots " penis » et « vagina » qui ressortent du continuum à côté d'autres leitmotivs comme "said she couldn't remember ", cherche à produire un effet presque hypnotique, accentuant à l'extrême la défamiliarisation du propos et la tension entre banalité et étrangeté. Il en résulte que la performance se recentre fortement sur la figure de l'auteur, sur la voix de Vanessa Place (une voix placée, prenante, assez bien timbrée, plutôt basse), sur son allure, sur son maintien corporel ; en fin de compte, c'est elle qui retient l'attention. Cette tension entre lecture et performance, textualité et auctorialité, me paraît un enjeu important de la poésie conceptuelle. 


\section{L'appropriation et ses effets}

des discours sociaux; il ne s'agit pas seulement d'écriture. Commençons par remarquer que dans sa trilogie, Vanessa Place sait ce qu'elle fait. Il en allait de même pour Reznikoff, quelle que soit la différence de perspective. Il en va de même pour Kenneth Goldsmith quand il publie sa trilogie new-yorkaise (Weather, Traffic, Sports), car il s'agit d'objets et d'informations dont il est un usager quotidien, attentif et réfléchi. Tous sont fondés à traiter de ces sujets; leur appropriation est légitime. Mais on ne peut en dire autant de tous les artistes ou poètes conceptuels, ni de tous les gestes d'appropriation. Pour nous en tenir à Vanessa Place, il est remarquable qu'elle se soit fait violemment prendre à partie lorsqu'elle a entrepris de découper au format Twitter et de poster jour après jour le texte intégral de Gone with the Wind, ou lorsqu'en marge de ce travail elle a retranscrit phonétiquement, en usant du pentamètre typique de la tradition métrique anglaise, les paroles que Prissy, l'esclave noire, prononce dans le film de Victor Fleming au moment de l'accouchement de Mélanie :

Miss Scarlett, effen we kain git de doctah w'en Miss Melly's time come, doan you bodder

Ah kin manage. Ah knows all 'bout birthin.

Ain' mah ma a midwife? Ain' she raise me

ter be a midwife, too? Jes' you leave it

ter me. She warn't dar. Well'm, Dey Cookie say

Miss Meade done got wud early dis mawnin'

dat young Mist' Phil done been shot an' Miss Meade ${ }^{8}$ [...]

11 Divers auteurs et groupes ont accusé Vanessa Place de « réinscrire le racisme dans la chair de chaque descendant d'esclaves", et de faire carrière sur ce fonds (les mêmes pétitionnent d'ailleurs pour que le film de Victor Fleming soit interdit d'écran). Identifiée à une incarnation du white power, elle a été sanctionnée, désinvitée, exclue de certaines revues - et sans doute complimentée par des gens qu'elle détestait'. Une avanie semblable, mais de plus de conséquence puisqu'elle est allée jusqu'à des menaces physiques, alors que les représailles contre Vanessa Place ne sortaient pas de la sphère universitaire, est arrivée à Kenneth Goldsmith lorsqu'il s'est approprié le rapport d'autopsie de Michael Brown, le jeune noir tué par un policier blanc à Ferguson, Missouri, en $2014^{10}$. Le poème de Goldsmith est intitulé "The Body of Michael Brown », allusion au célèbre chant de la Guerre de Sécession, « John Brown's Body » ; il s'inscrit à la suite d'une série de "désastres" américains, parmi lesquels l'assassinat de Kennedy (Goldsmith 2013). Ce sont dans tous les cas des textes d'archives policières ou judiciaires, publiées ou librement accessibles au public, retranscrits intégralement et mot pour mot (nonobstant quelques rares interventions rhétoriques, non dépourvues de coquetterie ou de provocation $\left.^{11}\right)$.

Dans ces débats typiques de la scène américaine, deux arguments importants sont avancés. Le premier est celui de l'appropriation. Goldsmith ou Place publient sous leur nom d'auteurs des textes qui sont disponibles matériellement (le plus souvent sur la Toile) et juridiquement (on peut le supposer). Dans certains cas, il est inévitable qu'ils entrent en concurrence avec des revendications, éthiques, politiques ou symboliques, de propriété. Sans doute Goldsmith a-t-il le mérite de poser avec netteté une question telle que : à qui appartient le corps de Michael Brown? Il n'en reste pas moins qu'il a mis son 
nom sur ce corps, et qu'il a dit à sa manière : ceci est à moi. Or Goldsmith, à la différence de situationnistes comme Debord, qui s'étaient fait du détournement une spécialité, ne situe pas son geste dans une critique générale de la propriété. Il n'est même pas évident qu'il ait voulu lui donner une signification militante, plutôt que d'en faire un exemple d' uncreative writing (chez Vanessa Place, la signification politique du geste est plus ouvertement assumée : il s'agit bien de travailler des documents et non de produire des textes $\left.{ }^{12}\right)$. Quitte à passer pour politiquement correct, je dirai qu'on peut comprendre que la communauté noire se soit sentie atteinte par la performance de Goldsmith. Elle avait d'autant plus de raison de l'être - c'est le second argument - que le geste fait entrer le corps de Michael Brown dans l'économie de marché. Avec ce corps, de la façon la plus littérale qui soit, Goldsmith (qui est dans sa partie un homme célèbre) a fait de l'argent. L'entrée dans les circuits de l'art n'est pas seulement légitimante, elle est aussi rémunératrice. Le modèle revendiqué des poètes conceptuels, ce sont des artistes conceptuels comme Sol LeWitt ou Andy Warhol, qui ont fait fortune avec leurs idées.

On objectera que la propriété, qui est ici un enjeu central, n'est pas celle du texte. Ce qui est en question n'est pas la pratique de la retranscription, comme dans le cas du plagiat littéraire ou du copier-coller en milieu universitaire. Goldsmith, comme la Bruyère avant lui (mais sans en tirer les mêmes conclusions) affirme que tout a été dit, que tous les discours sont disponibles, et qu'il appartient à tous d'en faire usage de toutes les manières possibles. Il nous délivre, et c'est certainement un apport positif et même une conquête, de l'exigence d'originalité et de l'anxiety of influence qui en est la contrepartie inévitable. Mais on peut estimer que s'il était vraiment conséquent, il devrait disparaitre dans ses actes et suivre l'exemple de Paul Nougé : «J'aimerais assez, que ceux d'entre nous dont le nom commence à marquer un peu, l'effacent ${ }^{13} "$ ([1929] 1980: 79). Ses gestes deviendraient-ils pour autant invisibles? Il est paradoxal que l'art conceptuel ait pour conséquence non l'avènement du concept, mais le triomphe de l'auteur, à qui la disparition de l'œuvre laisse tout loisir de tirer la couverture à lui. Goldsmith l'assume franchement, et dans le rôle d'un Playboy of the Western World version 2.0, il déploie un talent impressionnant. Le style de performance de Vanessa Place, ainsi que les photos où elle se met en scène (et dont la séduction et l'arrogance ne sont pas absentes) montrent qu'elle a aussi compris la leçon.

\section{Le concept et l'œuvre}

Dans ce domaine il est facile, et tentant, de faire n'importe quoi. C'est pourquoi je trouve important, comme je le disais plus haut, que l'auteur sache ce qu'il fait: soit qu'il ait une compréhension intime du fond de l'affaire, comme Vanessa Place dans le cas de Statement of facts (mais peut-être pas au même degré dans le cas de Gone with the Wind); soit que, comme dans Traffic, il n'y ait pas de fond autre que l'infra-ordinaire, celui de l'expérience absolument commune. Cette compréhension se marque évidemment dans le choix des éléments retenus, et le cas échéant dans leur agencement. C'est à ce niveau que se retrouve quelque chose qui s'apparente à l'autonomie de l'œuvre. La trilogie de Place, qu'elle intitule Tragodia, se réfère de manière explicite à des modèles rhétoriques et littéraires, qui constituent le cadre dans lequel son matériau est présenté à nouveaux frais - reframed -, et dans lequel il prétend devenir poème. Insistons sur le fait que le matériau retranscrit n'est en réalité accessible que dans ce cadre et sous cette forme; qu'il est en pratique impossible d'en prendre connaissance dans son ensemble et 
d'apprécier les opérations de sélection et de redistribution effectuées. On peut en dire autant pour le caractère verbatim, mais je crois que sur ce point nous n'avons aucune raison de ne pas croire les auteurs sur parole; tricher serait trop simple. Les travaux de Fiona Mc Mahon (2011) sur Reznikoff ont montré comment celui-ci travaillait, et le type d'interventions (coupures, réagencements) que dans certains cas il pratiquait, avec beaucoup de discrétion et de discernement. Mais lui seul aura lu ces milliers de pages d'archives dont il a fait Testimony, et il en va de même pour Vanessa Place dans ces livres qui touchent directement à l'exercice de son métier : leur œuvre vaut en tant que telle, à prendre ou à laisser.

La consistance de l'œuvre, en dépit des déclarations fracassantes sur l'écriture non créative, demeure une pierre de touche de la valeur de cet art conceptuel. L'œuvre doit s'imposer en tant qu'entité globale, mais aussi par sa réalisation, c'est-à-dire, puisqu'il s'agit de poésie, par son rapport au langage. Sur le plan global, ce qui compte est le rapport entre l'idée et le livre, et la manière dont l'une est incarnée - embodied - dans l'autre. Comme la lecture exhaustive et continue est à peu près impossible (à quoi bon lire Traffic ou Day d'un bout à l'autre, sinon pour éprouver la longueur du temps, comme dans la salle d'attente du dentiste?) on procède nécessairement par un va-et-vient entre une considération du concept, une appréciation de la forme d'ensemble (le format, le poids, le papier même), et des prélèvements de détail. Ici nous nous trouvons à notre affaire. Si Statement of facts est un poème, on doit pouvoir le soumettre avec profit à une procédure de close reading, comme on ferait pour un poème d'Emily Dickinson. Je m'y suis essayé, et le résultat est d'autant plus significatif à mes yeux que le texte n'a commencé à me paraitre intéressant que quand je l'ai recopié, ce qui m'a permis d'éviter de le qualifier ("style de procès-verbal », etc.) sans l'avoir lu-comme si le texte, dans son ordre, était un prévenu en attente de jugement, et qu'il faut traiter avec équité. C'est au prisme d'une lecture fine, mais exempte de préjugés quant à la «diction" (je n'ose imaginer ce que Genette en aurait pensé ), que le texte prend consistance et commence à produire ses effets, qui sont, me semble-il, ceux d'une prise de conscience et d'une critique du jugement. Avec cette lecture fine, le style de performance peut entrer en dialogue : c'est à cette condition seulement que les deux s'équilibrent.

Il faut tenir compte, d'autre part, du fait que je découvre ces statements dans le texte de Vanessa Place, même si la question, la thématique, le style de procès-verbal me sont relativement familiers. Il n'en va pas de même lorsque je relis Gone with the Wind ou On the Road de Kerouac, récrits ligne à ligne (par Vanessa Place, pour le premier) ou page à page (par Simon Morris, pour le second ${ }^{14}$ ) au fil des jours. Ce qui est recadré dans ce cas, c'est moins le texte que la lecture elle-même, si bien qu'il est impossible d'en parler sans avoir fait l'expérience de cette lecture dans la durée et éprouvé les effets que cette feuilletonisation radicale fait subir au roman. Travailler en lecture fine sur un échantillon n'a évidemment aucun sens : c'est pourquoi, faute d'expérience, je n'en parlerai pas ici.

On comprend donc que la question du close reading se soit invitée dans le débat américain sur la poésie conceptuelle. Dans le chapitre consacré à Kenneth Goldsmith de son livre Unoriginal Genius, où elle se livre à une défense et illustration de la poésie conceptuelle, Marjorie Perloff soutient l'idée que les livres de Goldsmith ne doivent pas être considérés comme une simple application du concept qui les régit - en un mot, qu'ils doivent être lus. Les manifestes de Goldsmith obéissent, dit-elle, à une rhétorique paradoxale. Focalisés sur le concept, ils ne fournissent pas une description adéquate des œuvres elles-mêmes: Goldsmith ne fait pas exactement ce qu'il dit, et nous n'avons pas à le croire sur parole 
lorsqu'il se définit comme « the most boring writer that has ever lived ». Voici en effet ce qu'il dit :

Conceptual writing is more interesting in a thinkership than a readership. Readability is the last thing on this poetry's mind. Conceptual writing is good only when the idea is good; often, the idea is much more interesting than the resultant text. (cité par Perloff 2010 : 201)

Les thèses de Goldsmith sur le conceptualisme transposent presque mot pour mot celles de Sol LeWitt, auxquelles il se réfère en s'accusant de les avoir plagiées. C'est ici que l'on voit ce qu'implique l'expression de "poésie rétinienne" avancée par Vanessa Place. Goldsmith pourrait la reprendre à son compte: l'un de ses arguments est que le développement du numérique place la poésie dans une situation équivalente à celle que la peinture a connue au moment de l'apparition de la photographie. Mais cette expression de " poésie rétinienne » est une métaphore, dont la cohérence et les implications ne vont pas de soi. Dans l'art conceptuel, deux systèmes sémiotiques au moins sont mobilisés : le « concept » est formulé avec les moyens d'une langue naturelle, mais sa mise en œuvre passe par une réalisation plastique, bidimensionnelle ou tridimensionnelle. Il est impossible de générer une œuvre à partir de sa consigne sans un travail de réalisation qui implique, en lui-même, un nombre considérable de décisions et de choix. Les notes rédigées par David Schulman, le dessinateur qui fut chargé d'exécuter le "concept " formulé par Sol LeWitt pour une peinture murale du Guggenheim Museum en 1971, fournissent une évocation suggestive des multiples difficultés rencontrées, difficultés qui finirent par se résoudre dans une intériorisation du programme, proche des techniques de méditation du yoga: détente complète, vide de l'esprit, concentration du corps (Goldsmith 2011a: 134-135). La poésie conceptuelle, au contraire, va du langage au langage : c'est la raison pour laquelle Goldsmith peut poser une équivalence entre l'idée et l'œuvre, et affirmer qu'en termes d'usage, la première remplace avantageusement la seconde.

Or la substitution de l'auteur à l'œuvre est conforme aux attentes de la société du spectacle, de même que la substitution de l'idée à l'œuvre satisfait aux modes de transmission de l'information dans l'espace numérique : les idées sont plus simples, plus « partageables » et plus « aimables » que les œuvres. Le risque est que le poète ne soit plus que l'homme-sandwich et le maquereau de ses idées. Cette objection a été soulevée à juste titre par Ron Silliman, un poète pourtant peu suspect de conservatisme. Dès lors que le projet est conçu de manière à empêcher toute autonomie de l'œuvre, dit-il, il aboutit nécessairement au culte de l'artiste devenu lui-même œuvre d'art : « Kenny Goldsmith's actual art project is the projection of Kenny Goldsmith» (cité par Perloff $2010: 203$ ). Le talent de performeur de Goldsmith (comme on l'a vu, Vanessa Place n'est pas en reste) accroît encore cette prégnance d'une auctorialité visible. Le concept, la performance et l'auteur forment ainsi une sorte de triangle maléfique où la substance de l'art menace de s'engloutir.

\section{Lecture et légitimation}

C'est pourquoi le close reading, qui est entièrement focalisé sur l'œuvre, fait figure de contrepoison. Perloff s'emploie à montrer, en soumettant Traffic à l'épreuve d'une lecture fine, les complexités cachées du livre, ses incertitudes (quel est le "big weekend holiday " dont il est question? à quel moment de ce week-end se trouve-t-on?), ses incohérences et 
absurdités comiques, les schèmes narratifs qui apparaissent et se défont: une connaissance de la réalité prend forme à travers son analyse, et cette réalité (c'est-à-dire une vision «objectiviste» du New York actuel) n'est accessible qu'au prix d'une immersion dans la durée de la lecture, mais aussi d'une activité de comparaison et de recoupement dont une écoute continue sur une durée équivalente du bulletin-source n'offrirait aucunement l'équivalent. Cette lecture interne se double d'une lecture externe, où le traitement informatif des accidents est comparé, de manière très suggestive, à ce que font de ce thème J.C. Ballard (et David Cronenberg) dans Crash, et Jean-Luc Godard dans Week-end.

Je n'ai pas vérifié par moi-même les allégations de Perloff, mais l'examen attentif d'autres échantillons me conduit aux mêmes conclusions. La manière, par exemple, dont est mis en page le texte du New York Times dans Day, n'a rien d'automatique; on pourrait la programmer, mais il est évident que l'auteur a procédé de manière inductive, empirique, et que d'autres choix étaient possibles. Lui-même en convient :

I could take any one of my books - say, Day - and devise a recipe: "Retype a day's edition of the New York Times from beginning to end, working your way across the page, left to right. Retype any letter in the paper, making no distinction between editorial and advertising." Surely your choices - the way you make your way through the paper, how you choose to break the lines, etc., - will be different than mine, making for a completely different work. (Goldsmith 2011a : 129)

On peut en dire autant des exemples les plus convaincants que rassemble l'anthologie de Goldsmith et Craig Dworkin, Against Expression (2011) : parmi ceux-ci je mentionnerai Sally Alatalo, Caroline Bergvall, Christian Bök, Douglas Huebler, Alexandra Nemerov, Julia Spahr. Dans tous ces cas, on voit clairement que c'est le rapport entre conception et textualisation qui est productif. J'entends « textualisation » du point de vue du lecteur : il y a «travail» textuel, mais dans certains cas, c'est le lecteur qui fait ce travail-typiquement, dans la compilation par Caroline Bergvall des deux cents traductions anglaises du premier tercet de La Divine Comédie. Dans le poème d'Alexandra Nemerov, «First my Motorola », il est impossible de séparer l'idée qui consiste à recadrer une journée ordinaire à partir de la liste des marques avec laquelle "je » entre en relation, l'autoportrait d'une femme moderne (jeune, urbaine, branchée, etc.) qui s'en dégage, la mise en évidence critique du formatage consumériste, et le poème lui-même, avec sa litanie chantante des "then my» dans le vers libre court et le kaléidoscope sonore des marques :

First, my Motorola

Then my Frette

Then my Sonia Rykiel

Then my Bulgari

Then my Asprey

Then my Cartier

Then my Kohler

Then my Brightsmile

Then my Cetaphil

Then my Braun

Then my Brightsmile

Then my Kohler

Then my Cetaphil

Then my Bliss [...]

(Against Expression 2011 : 457) 
aux propositions de Christian Bök, elles sont aussi fascinantes et aussi baroques d'un point de vue conceptuel (l'idée d'implanter un texte dans une bactérie à partir d'une littéralisation du codage ADN, dans Xenotext Experiment) que d'un point de vue textuel : à elle seule la perception par l'œil des chapitres monovocaliques d'Eunoia, le premier en $a$, le second en $e$, et ainsi de suite, avec les variations topiques et modales qui en sont la conséquence, suscite un véritable choc. Il ne fait aucun doute que ces œuvres existent par elles-mêmes.

n reste pas moins que l'analyse de Perloff a un fort pouvoir de légitimation. En proposant une lecture lyrique de textes ouvertement anti-lyriques, elle les inscrit dans une longue tradition de la poésie occidentale où le renouvellement se fait par une contestation des formes ; elle les intègre à une histoire de l'avant-garde, et valide par voie de conséquence non seulement les pratiques de retranscription ou de recadrage sous leurs multiples formes, mais le geste même de réappropriation par lequel l'auteur prétend faire du neuf - make it new. Elle légitime Goldsmith ou Place en tant que poètes - ce qui, intuitivement, ne va tout de même pas de soi - avec les prédicats d'universalité qui s'attachent traditionnellement à ce terme : une universalité que ses détracteurs noirs ne se font pas faute de présenter comme l'apanage de l'homme blanc, c'est-à-dire de celui qui n'a pas à se définir par sa race. Et elle les légitime comme poètes d'avant-garde, les inscrivant dans le sens de l'histoire. Dans le même temps, Perloff traite de "populistes » les poètes afro-américains que Cary Nelson a fait entrer dans son Anthology of Modern American Poetry (2000), en faisant bon marché des conditions historiques dans lesquelles ces « voix » ont pu émerger et de leur rapport spécifique à la tradition (en particulier à la poésie victorienne). Ses critères d'évaluation essentialistes («qu'est-ce que la poésie?»), esthétiques («qu'est-ce que la bonne poésie?») et historiques («qu'est-ce que l'avantgarde?») l'amènent à considérer les poètes conceptuels comme les incarnations modernes du génie - un mot dont l'apparition dans ce contexte a de quoi faire sursauter.

L'appartenance à l'avant-garde de nos deux auteurs n'est pas contestable. Pour Kenneth Goldsmith, figure clé de cette poésie conceptuelle, elle est au fondement de sa culture, comme le montre la continuité proposée par la séance à la Maison-Blanche où il enchaîne sur le thème du pont de Brooklyn des extraits de Walt Whitman, de Hart Crane et de son propre Traffic ${ }^{15}$; elle est inscrite dans la genèse de son œuvre, avec ses débuts par la poésie concrète ; elle a pris une ampleur patrimoniale avec la création du site UbuWeb, qui est une mémoire vivante, intermédiale, des avant-gardes occidentales. Sa poésie conceptuelle hérite du modernisme, mais dans la filiation de Reznikoff plutôt que dans celle de Pound. La situation de Vanessa Place est plus ambiguë. On perçoit dans son travail une tension entre deux conceptions de l'avant-garde (la même que chez Marjorie Perloff) : le manifeste qu'elle donne à Poetry, " No more ", refuse l'héritage moderniste tout en mimant le geste qui l'avait inauguré. Mais son œuvre ne se limite pas à la poésie conceptuelle. Vanessa Place est aussi au sens plein du terme (celui du creative writing) un auteur. Avant Statement of facts, elle a publié La Medusa (2008), roman " polyphonique » sur Los Angeles, et Dies: A Sentence (2005), remémoration d'un soldat mourant formée d'une seule phrase de cinquante mille mots. Ces livres se différencient nettement de compilations comme Weather ou Traffic. La grande composition éclatée, la phrase-livre et le flux de conscience sont des marqueurs majeurs du modernisme, que Vanessa Place 
réactualise après le scepticisme post-moderne et en rupture avec lui. Il $\mathrm{y}$ a là de quoi expliquer, et peut-être justifier, ce qui peut sembler un déport vers une auctorialité autosuffisante, devenue principe et fin de la poésie.

Je me permets, en conclusion, de résumer mon point de vue : il me semble important que les œuvres conceptuelles soient soumises au même protocole de lecture que les œuvres modernistes, en tenant compte de leur spécificité : c'est-à-dire à une mise en rapport du concept, de l'œuvre dans sa globalité (du livre, de la série de livres) et du texte. Le close reading conserve tous ses droits. L'auctorialité doit être prise en considération sans être sacralisée, maintenue dans l'ordre où elle s'exerce, et en même temps comprise comme un facteur constitutif de l'œuvre - comme l'exemple de Warhol le montre avec éloquence. C'est la seule manière que nous ayons de maintenir au sein d'une telle production un jugement de valeur, et de distinguer le meilleur du médiocre (quitte à nous tromper de temps en temps). Le travail de Marjorie Perloff est sur ce point un exemple à suivre. Gardons-nous simplement, comme elle semble le faire dans sa démarche militante et parfois sectaire, d'identifier cette poésie à la poésie : n'oublions pas qu'il y a plusieurs demeures dans la maison du Père.

\section{BIBLIOGRAPHIE}

Bernstein, Charles, 2010, All the Whiskey in Heaven, New York, Farrar, Strauss \& Giroux.

Dworkin, Craig et Goldsmith, Kenneth (éd.), 2011, Against Expression. An Anthology of Conceptual Writing, Evanston, Northwestern University Press.

Goldsmith, Kenneth, 2011a, Uncreative Writing, New York, Columbia University Press.

Goldsmith, Kenneth, 2011b, The White House Poetry Reading, 11 mai 2011, [En ligne], https:// www.youtube.com/watch?v=hMSvrIPhA4Y, consulté le 14 juin 2018.

Goldsmith, Kenneth, 2013, Seven American Deaths and Desasters, New York, powerHouse Books.

Keene, John, 2015, « On Vanessa Place, Gone With the Wind, and the Limit Point of Certain Conceptual Aesthetics ", Atticus Review, [En ligne], https://atticusreview.org/on-vanessa-placegone-with-the-wind-and-the-limit-point-of-certain-conceptual-aesthetics-2/, consulté le 14 juin 2018.

Mc Mahon, Fiona, 2011, Charles Reznikoff. Une poétique du témoignage, Paris, L'Harmattan.

Mehigan, Joshua, 2013, « Make Make it New New », Poetry, [En ligne], https://atticusreview.org/ on-vanessa-place-gone-with-the-wind-and-the-limit-point-of-certain-conceptual-aesthetics-2/, consulté le 14 juin 2018.

Nelson, Cary, 2000, Anthology of Modern American Poetry, Oxford, Oxford University Press.

Nougé, Paul, 1980, Lettre à André Breton, 2 mars 1929, dans Paul Nougé, Histoire de ne pas rire, Lausanne, L'Âge d'homme.

Perloff, Marjorie, 2010, Unoriginal Genius: Poetry by Other Means in the New Century, Chicago, University of Chicago Press. 
Perloff, Marjorie, 2012, « Poetry on the Brink. Reinventing the Lyric », Boston Review, [En ligne], http://bostonreview.net/archives/BR37.3/marjorie_perloff_poetry_lyric_reinvention.php, consulté le 14 juin 2018.

Peronny, Nathalie, 2010, Exposé des faits, Alfortville, Ere.

Place, Vanessa, 2011, « The Death of the Text, Kenneth Goldsmith at the White House », [En ligne], https://www.poetryfoundation.org/harriet/2011/05/the-death-of-the-text-kennethgoldsmith-at-the-white-house/, consulté le 14 juin 2018.

Place, Vanessa, 2007, Dies: A Sentence, Los Angeles, Les Figues Press.

Place, Vanessa, [2005] 2008, La Medusa, Tuscaloosa, FC2, University of Alabama Press.

Place, Vanessa, 2009, « Miss Scarlett », Poetry, juillet-août 2009, [En ligne], https:// www.poetryfoundation.org/poetrymagazine/poems/52693/miss-scarlett, consulté le 14 juin 2018.

\section{NOTES}

1. Ma réflexion sur la poésie conceptuelle américaine s'est appuyée principalement sur les ouvrages suivants : Goldsmith (2011), Perloff (2010), Dworkin et Goldsmith (2011). Je remercie Abigail Lang pour sa relecture et pour les précisions qu'elle m'a apportées.

2. Poetry, Chicago, mars 2013 (https://www.poetryfoundation.org/poetrymagazine/poems/ detail/56142). Les autres contributeurs de cette rubrique sont Joshua Mehigan, Reginald Dwayne Betts (un Afro-Américain) et Jill Alexander Essbaum.

3. C'est le constat que fait Joshua Mehigan dans sa contribution à la même livraison de Poetry, «Make Make it New New» (https://www.poetryfoundation.org/poetrymagazine/articles/ detail/69941). Il est partagé par Marjorie Perloff, dont les orientations sont pourtant bien différentes : « Whatever the poet's ostensible subject - and here identity politics has produced a degree of variation, so that we have Latina poetry, Asian American poetry, queer poetry, the poetry of the disabled, and so on - the poems you will read in American Poetry Review or similar publications will, with rare exceptions, exhibit the following characteristics: 1) irregular lines of free verse, with little or no emphasis on the construction of the line itself or on what the Russian Formalists called "the word as such"; 2) prose syntax with lots of prepositional and parenthetical phrases, laced with graphic imagery or even extravagant metaphor (the sign of "poeticity"); 3) the expression of a profound thought or small epiphany, usually based on a particular memory, designating the lyric speaker as a particularly sensitive person who really feels the pain, whether of our imperialist wars in the Middle East or of late capitalism or of some personal tragedy such as the death of a loved one. " (Marjorie Perloff, "Poetry on the Brink. Reinventing the Lyric ", Boston Review, mai-juin 2012: http://bostonreview.net/archives/BR37.3/ marjorie_perloff_poetry_lyric_reinvention.php).

4. En témoigne la publication en 2010 de l'anthologie personnelle de Charles Bernstein, All the Whiskey in Heaven, par Farrar, Strauss \& Giroux - l'équivalent de la collection «Blanche» de Gallimard.

5. Il s'agit du premier volume d'une trilogie : Tragodia 1: Statement of Facts ; Tragodia 2: Statement of The Case ; Tragodia 3: Argument (Los Angeles, Insert Blanc Press, 2010, 2011). Le premier volume a été traduit par Nathalie Peronny : Exposé des faits, Alfortville, Ere, 2010.

6. Extrait cité dans Dworkin et Goldsmith (2011 : 491) ; ibid., pour les citations suivantes.

7. On peut consulter sur Pennsound sa performance du 24 mars 2011 à Kelly Writer's House, Université de Pennsylvanie (http://writing.upenn.edu/pennsound/x/Place.php). 
8. Vanessa Place, «Miss Scarlett », Poetry, juillet-août 2009. Le poème est accompagné d'une note explicative : «Taken from Prissy's famous scene in the movie version of Gone with the Wind, Place phonetically transcribes the "unreliable" slave's words, which are then set in Miltonic couplets. Through the simple act of transcription, Place inverts our relationship to Margaret Mitchell's best-selling and beloved American epic by prioritizing the formal aspects of language over Mitchell's famous narrative. With this deconstructive move, Place illuminates the many subtexts embedded in the text concerning plays of power, gender, race, and authorship. By ventriloquizing the slave's voice as well as Mitchell's, Place also sets into motion a nexus of questions regarding authorship, leading one to wonder: who is pulling whose strings? » (https:// www.poetryfoundation.org/poetrymagazine/poems/detail/52693).

9. On trouve un bon commentaire de cette affaire dans l'article de John Keene, «On Vanessa Place, Gone With the Wind, and the Limit Point of Certain Conceptual Aesthetics ", Atticus Review, mai 2015 (https://atticusreview.org/on-vanessa-place-gone-with-the-wind-and-the-limit-pointof-certain-conceptual-aesthetics-2/). Sur le fil Twitter dédié à cette œuvre, Vanessa Place affiche comme avatar l'image de Hattie Mc Daniel, qui jouait Mammy dans le film de Fleming; le « recadrage » de cette icône, qui est un stéréotype majeur, a contribué à agiter les esprits.

10. Goldsmith a donné lecture de son poème dans la conférence «Interrupt 3 » de l'Université Brown en mars 2015. Pour un exposé détaillé des circonstances et du débat qui a suivi, voir l'article de Jillian Steinhauer sur le blog Hyperallergic : https://hyperallergic.com/190954/.

11. Le poème se termine sur la phrase suivante: "The remaining male genitalia system is unremarkable ", alors que cette phrase n'est pas la dernière du rapport d'autopsie. On peut trouver le procédé d'un goût douteux, mais il est certain que Goldsmith met le doigt sur un des ressorts les plus durables de toute l'histoire interraciale des États-Unis, la survirilité supposée de l'homme noir.

12. Je remercie Franck Leibovici pour les suggestions qu'il m'a faites sur ce point.

13. Nougé devrait figurer en bonne place dans une anthologie telle que Against Expression, mais son nom n'a pas franchi l'Atlantique.

14. Simon Morris s'y est livré en 2009 dans un blog intitulé : Getting inside Kerouac's Head (http:// gettinginsidejackkerouacshead.blogspot.fr/) ; voir Goldsmith (2011a : 150 et suiv.).

15. Kenneth Goldsmith, The White House Poetry Reading, 11 mai 2011 (https://www.youtube.com/ watch?v=hMSvrIPhA4Y). Voir le commentaire de Vanessa Place, « The Death of the Text, Kenneth Goldsmith at the White House ", sur le blog de la Poetry Foundation (https:// www.poetryfoundation.org/harriet/2011/05/the-death-of-the-text-kenneth-goldsmith-at-thewhite-house/).

\section{RÉSUMÉS}

La poésie conceptuelle est une des plus remarquables manifestations actuelles des avant-gardes américaines. En prenant l'exemple de deux de ses représentants majeurs, Kenneth Goldsmith et Vanessa Place, l'article propose trois sujets de réflexion : la relation, filiation ou rupture, de cette poésie avec la tradition moderniste; les implications sociales et politiques du geste d'appropriation de discours qui la constitue ; la possibilité de considérer les œuvres issues de ces pratiques «non créatives » comme des textes susceptibles non seulement d'une compréhension conceptuelle, mais d'une lecture attentive à la forme, telle que le close reading en a posé les principes. 
Conceptual poetry is one of the most significant expression of American avant-gardes, Kenneth Goldsmith and Vanessa Place being their prominent representatives. This paper aims to question three issues: the relationship, filiation or rupture, of this kind of poetry with the modernist tradition; the social and political meaning of the appropriation of their body of discourse; the status of "non-creative" works when confronted to other interpretations than conceptual ones, such as form analysis and "close reading."

INDEX

Mots-clés : poésie, poésie conceptuelle, avant-gardes, performance, modernisme, Reznikoff (Charles), Goldsmith (Kenneth), Place (Vanessa)

Keywords : poetry, conceptual poetry, avant-gardes, performance, modernism, Reznikoff (Charles), Goldsmith (Kenneth), Place (Vanessa)

\section{AUTEUR}

MICHEL MURAT

Université Paris-Sorbonne, Cellf 